Binghamton University

The Open Repository @ Binghamton (The ORB)

The Society for Ancient Greek Philosophy Newsletter

$11-28-1958$

\title{
Plato's Hypothesis and the Upward Path
}

Thomas G. Rosenmeyer

University of Washington, Seattle

Follow this and additional works at: https://orb.binghamton.edu/sagp

Part of the Ancient History, Greek and Roman through Late Antiquity Commons, Ancient Philosophy Commons, and the History of Philosophy Commons

\section{Recommended Citation}

Rosenmeyer, Thomas G., "Plato's Hypothesis and the Upward Path" (1958). The Society for Ancient Greek Philosophy Newsletter. 236.

https://orb.binghamton.edu/sagp/236

This Article is brought to you for free and open access by The Open Repository @ Binghamton (The ORB). It has been accepted for inclusion in The Society for Ancient Greek Philosophy Newsletter by an authorized administrator of The Open Repository @ Binghamton (The ORB). For more information, please contact ORB@binghamton.edu. 
Thomas G. Posenmeyer

University of Washington

Seattle, Washington

Plato's Hypothesis and the Upward Path

Richard Robinson, in his stimulating and searching book on Plato's Earlier Dialectic, has a section entitled "Hypothesizing a Higher Hypothesis."I Like other recent scholars, he takes it for granted that in Plato's scheme of hypothetical reasoning as developed in the Phaedo and the Republic, the more general.

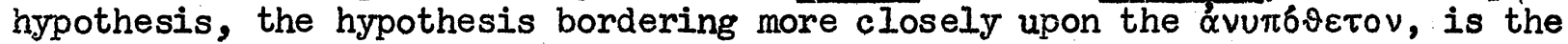
higher hypothesis. That is to say, in a deductive system, as the Latin base of the term indicates, the premises are thought of as located higher than the conclusions. In this, Robinson follows not only what he conceives to be Plato's own hints, but also the usage of Aristotle: "By 'upward' I mean the ascent to the more universal, by 'downward' the descent to the more particular." 2 In this footnote on terminology, Aristotle is talking about natrropial, predicates in syllogistic reasoning. It might at first be supposed that " $\alpha \omega$ and $u^{*} \tau \omega$ are references to the purely formal structure of the syllogism itself rather than to the structure of the reality signified by the terms of the syllogism. 3 But other passages make sufficiently clear that the reference is indeed to higher and lower Being. 4 For Aristotle, the movement of the syllogism is downward because we demonstrate certain conclusions from certain assumptions - often, paradoxically, called $x \varepsilon\{\mu \varepsilon v \alpha$ - of a more universal

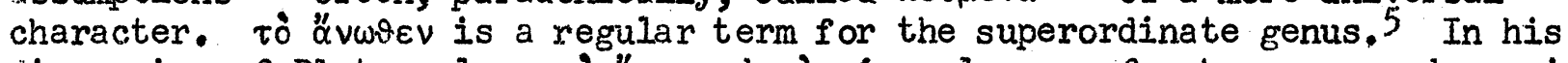
discussion of Plato, also, $\tau \dot{\alpha} \alpha \nu \omega$ and $\tau \dot{\alpha} x \alpha \tau \omega$ always refer to genus and species ${ }^{6}$ It is not surprising, therefore, that Aristotle's logical terminology is generally thought to be based on Plato's. The facts, however, do not entirely bear out this view. In this paper I shall attempt to show that Aristotle's logical terminology, ontologically conditioned or determined as it obviously is, contrasts with that of Plato, who, on the whole, with perhaps one exception, was very careful, not to introduce unwarranted ontological perspectives into matters of logic.?

To begin with, it will be convenient to note the implications of Platonic diaeresis. It is true that this operation of Platonic dialectic is not the same as the logical progress from premise to inference. Genus and species are ontological rather than logical terms. And yet it may be assumed that if Plato's logic anticipated the Aristotelian distinction between the higher and the lower, the same topographical perspective should also be apparent in the area studied by diaeresis. It will be all the more striking, then, if we can show that diaeresis does not make provision for a vertical hierarchy of Being, or, at least, that Plato's discussion does not suggest that such a hierarchy is reflected in the diaeretic procedure so as to shape it in its image.

Modern discussions of Plato's analysis via division and collection often refer to higher or superordinate and lower or subordinate $\gamma \varepsilon v \eta$. $\mu \xi \gamma\left\llcorner\sigma \tau \alpha\right.$ $\gamma^{k} \nu$ is sometimes rendered as "highest forms." And elegant stemmata are devised, 
spreading from the top of the page to the bottom, to reproduce the up- or downward movement of the dialectical process. But if we look closely at the relevant passages, both at those where Plato practices the technique of division and collection, and at those in which he states his method, we find that the directional implications of the process are either indifferent or horizontal. That is to say, to the extent that Plato conceives the diaeretic procedure as moving, it moves on one plane or level.

Here is a listing of the principal passages in question, with the key words, the topographical orientation implied, and occasional further comment.

Phaedrus 265D - 266C: diaeresis operates by dividing the entity to be analyzed into a left ( $\varepsilon \pi$ ' $\alpha \rho \iota \tau \varepsilon \rho \dot{\alpha} \tau \varepsilon \mu \nu \delta \mu \varepsilon \nu \circ \varsigma$ ) and a right part ( $\varepsilon_{\zeta} \tau \dot{\alpha}$ '́v $\delta \varepsilon \xi(\tilde{\alpha})$. Orientation: not vertical. Hence such terms as "sub-form" and "infima species" should be eliminated from the discussion of diaeresis passages. 9

Sophist 253: analysis consists of showing which $\gamma^{\varepsilon} v \eta$ associate with one

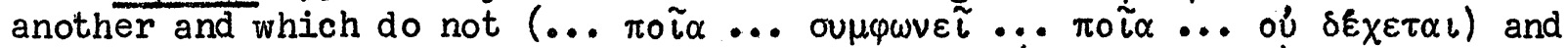
which $\gamma^{\varepsilon} \nu \eta$ have another "stretched through" them ( $\left.\delta \iota \alpha \tau \varepsilon \tau \alpha \mu \varepsilon \nu \eta \nu\right)$ so as to contain them ( $\pi \varepsilon \rho\llcorner\varepsilon \chi 0 \mu \varepsilon v a \varsigma)$. Orientation: indifferent.

Politicus 262A - 263B: analysis should operate by cutting through the middle $(\delta\llcorner\alpha \mu \varepsilon \sigma o v . .$. lहv $\alpha \iota \tau \varepsilon \mu \nu \circ \nu \tau \alpha \varsigma)$. Orientation: inconclusive.

Politicus 268C: analysis involves disengaging the item to be isolated

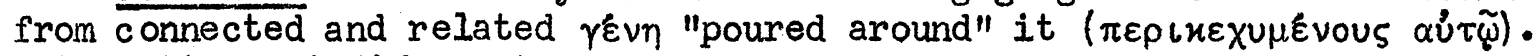
Orientation: indifferent.

Politicus 275E: the choice of the proper inclusive concept will allow us to "wrap up" ( $\pi \varepsilon \rho(น \alpha \lambda \theta \pi \tau \varepsilon(v)$ one species along with the others with which it constitutes a genus. Orientation: indifferent.

Politicus 285A-C: analysis requires orderly procedure, especially the grouping of separate elements into the one comprehensive genus to which they

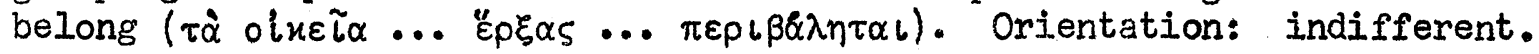

Philebus 16D - 18D: the analogy of grammatical and musical analysis shows that philosophical analysis must interpose various stages between the conception of the one and the conception of the infinite number containing

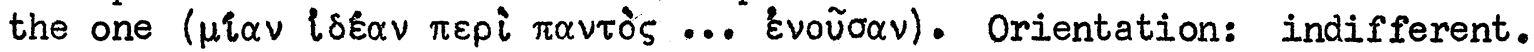

Sophist 265E-266A: In a progressive division of a $\gamma \varepsilon v 05$ into $2,4,8$, etc., parts, the division is made alternately by drawing a vertical line ( $(\alpha \tau \dot{\alpha} \pi \lambda \alpha \tau \circ \varsigma)$ and a horizontal line ( $\alpha_{\alpha} \alpha \dot{\alpha} \mu \tilde{n}$ nos). Orientation: indifferent.

Other references could be added, but the result would be the same, namely, that logical implication is regarded by Plato not as subsumption but either as containment - that is to say, the genus envelops the species - or as division - that is, the species constitutes the right or left half of the genus. The movement experienced in the transition from genus to species or, in the case of collection, from species to genus is not in a single instance characterized as a descent from or an ascent to the genus. 
One possible answer to this would be that the $\varepsilon^{\prime \prime} \delta \eta$ may not be organized in a vertical hierarchy, but that the Ideas of Plato's middle dialogues are,

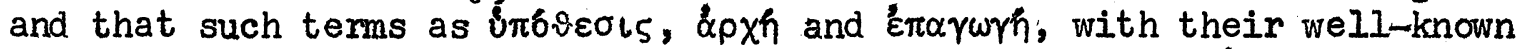
connotations, speak palpably for a vertical perspective. É $\pi \alpha \gamma \omega \gamma$ m may be dispensed with out of hand. Even Robinson, who insists that Plato practices $\varepsilon \pi \alpha \gamma \omega \gamma h$, admits that he is not aware of it as a logical procedure, and has in fact no term for it. Does Plato use induction? The boundary line separating induction from analogy is of course tenuous. But "all that business about cobblers and cleaners and cooks and doctors," as Callicles calls it, suggests that when Plato refers to practical reality, he appeals to some sort of intuition rather than the powers of reasoning. However that may be,

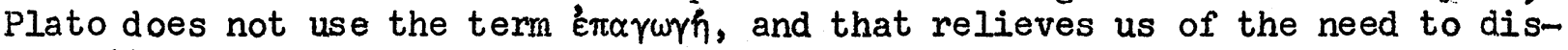
cuss it.

As for $i \pi 6 \vartheta \varepsilon \sigma \iota s$ and its near-equivalent $\alpha \rho x h$, the situation is very complex. Robinson himself has done much to shed light on the obscure standing of hypothesis in Plato. According to him, the case is the reverse of that obtaining in the matter of induction: "Plato discussed but rarely used the hypothetical method." Since, therefore, we have Plato's methodology but little of his application, obscurities and even inconsistencies in the former leave matters somewhat doubtful. This much, however, is clear. A hypothesis is something posited - cf. Aristotle's $u \varepsilon i \mu \varepsilon v o v$ - as a preliminary assumption, as a basis 10 for further logical operations which may either analyze the assumption itself or depend on it for the analysis of other assumptions. Normally the latter is the case, i.e., the hypothesis is a premise rather than a demonstrand. 11 Broadly speaking, then, "hypothesizing is positing with a view to future action." Often the assumption which forms the point

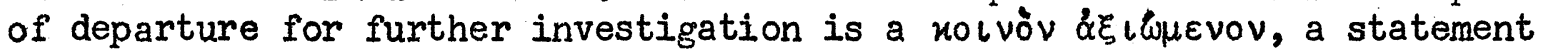
tacitly assumed to be true by all.

The important question which now arises is this: does the inb-part of the word i $\pi 6 \vartheta \varepsilon \sigma / 5$ signify that this basic assumption is conceived of as $1 \mathrm{y}-$ ing under and thus supporting the logical edifice constructed from it? Iet us call this the $U-(=$ under $)$ perspective, to contrast with the A- (= above) perspective whereby the assumption is conceived of as lying above the conclusion. Now on the face of it, it would seem likely that the ino points to a U-perspective. But according to Robinson and most Platonists that cannot be, for a hypothesis to be useful must refer to a higher reality than the propositions deduced from it. Hence Plato's hypothesis, like Aristotle's premise, and equally paradoxically so, must be pictured as vaulting above the conclusions and deductions which are suspended below it.

To render this unlikely notion palatable, Robinson undertakes, in the wake of Burnet, 12 to demonstrate that the first sense of hypothesis was intellectual, not architectural or physical in any way. 13 His reasoning is that if the word had ever "borne some such sense as 'physical foundation',

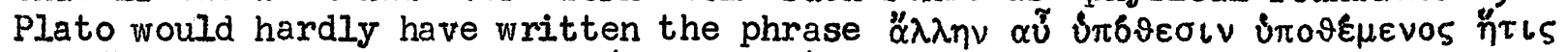

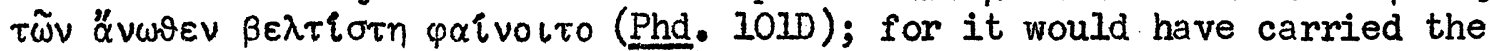
absurd suggestion of 'placing as base whatever base seemed best of those above'!" As we shall see later, Robinson's interpretation of the Phaedo passage, though commonly accepted, is not the only possible one. $\overline{A t}$ Republic 511B, where Plato does understand hypothesis in the sense of a 


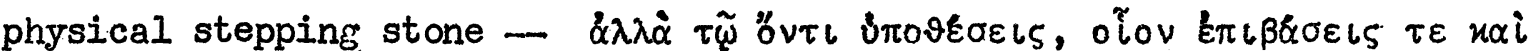
$\delta \rho \mu k \varsigma$... - Burnet and Robinson suspect a pun. But even granted that Plato is speaking humorously here, the passage proves that he felt the ino-part of the term strongly enough to allow his conception of logical procedure, at this point, to be guided by it or at least to make allowance for it.

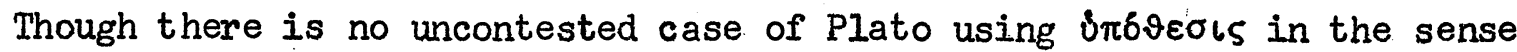
of "foundation," other writers supply us with the required supplementary information. Closest to that of Plato is, perhaps, the usage of the Hippocratic writers. For instance, the writer On Ancient Medicine (chs. 1; 13; 15) uses

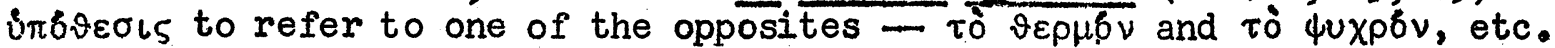
- alleged to determine health, as well as to the assumption of the operation of such an opposite. The spatial perspective is not entirely clear, but it cannot be doubted that these opposites should be ranged closely with the Empedoclean $\rho \iota \zeta 6 \mu \alpha \tau \alpha$, and as $\delta\llcorner\zeta 6 \mu \alpha \tau \alpha$ they would of course realize their inbfunction to the fullest.

Other writers who capitalize on the $\dot{\delta} \pi b$-force of the word $\delta \pi \delta \vartheta \varepsilon \sigma \iota s$ are Aeschines (3.76) and Polybius (15.35.2). But our clearest evidence for the dynamic implications of únóveoıs occurs in a popular text, and a simile, to boot. Demosthenes 2.10.5: "Just as a house and a ship and other such structures require the strongest foundations, so the $\alpha \rho \chi \alpha \ell$ and $\delta \pi 0 \vartheta \varepsilon \sigma \varepsilon \downarrow s$ of actions must be true and just." Here there can be no doubt; a hypothesis is analogous to the foundation of a house, not to its roof. And this, I suggest, was the natural significance of the word ư $\pi \vartheta \varepsilon \sigma \iota s$, before its meaning was obscured in a manner to be described directly: a foundation upon which to erect a superstructure of some sort, a broad basis on which things of lesser extent but greater concreteness are supported. 14

Logical progression, therefore, naturally leads from the hypothesis below to the inferences above. But there is another way of looking at logical progression. It may be pictured as leading forward horizontally from the start to the finish of a sentence or argument. This perspective is well illustrated

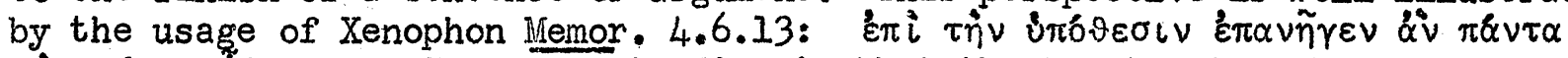
$\tau o v \lambda \delta$ yov $\omega \delta \varepsilon \ldots$. The understanding is that the hypothesis, the subject or foundation of the discussion, had been voiced, or should have been voiced, at the beginning of the conversation. A "getting down to fundamentals" is, therefore, a "going back" to the beginning. Here the horizontal advance of the discussion has imposed its stamp on the topography of $\dot{\delta} \pi \hat{v} \varepsilon \sigma \mathrm{c}$. In most cases it is a relatively simple matter to distinguish between this perspective and the perspective which mirrors the structural concept outlined above.

After these few preliminary remarks, we must now turn to some of the passages in which Plato exemplifies his understanding of inoveols. Again our list is representative rather than exhaustive. As before, I shall state the references, cite crucial words, and indicate the contribution of the passage to the subject under discussion. The actual word ífóvols does not occur in all of the passages cited. Sometimes apx takes its place; sometimes there is no technical term to be found. Not all of the passages describe what we would term a logical procedure. But the process envisaged is always the same: the movement from general assumption to specific conclusion. It is this movement which according to the usual interpretation should be a movement ä $\nu \omega \vartheta \varepsilon v$ hớ $\tau \omega$. 


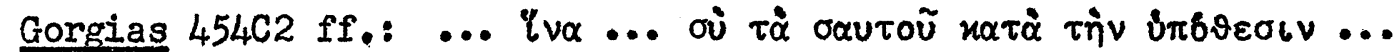
$\pi \varepsilon p \alpha$ ใทुs. The perspective is undetermined.

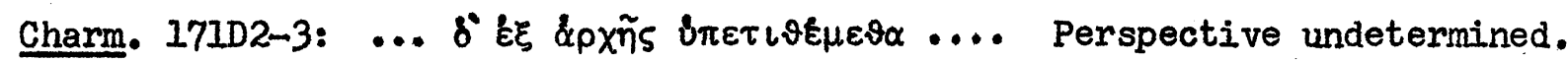

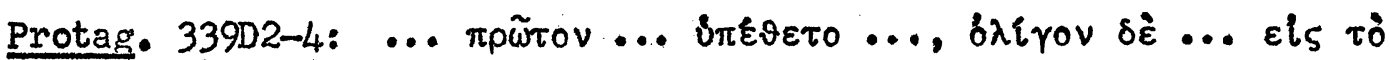
$\pi \rho 6 \sigma \vartheta \varepsilon v \pi \rho \circ \varepsilon \lambda \vartheta \iota \nu . .$. Perspective horizontal.

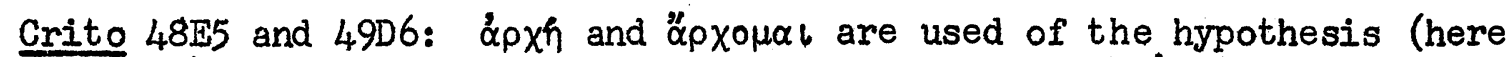
not so called) from which further statements are deduced. \&pх斤 is seen as the beginning of a $\delta \delta 6 \varsigma$. Perspective undetermined.

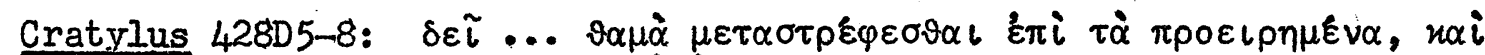

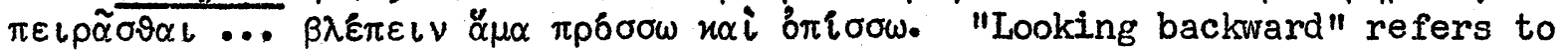
revising prior agreements, "looking forward" to inferring or deducing results. Perspective horizontal.

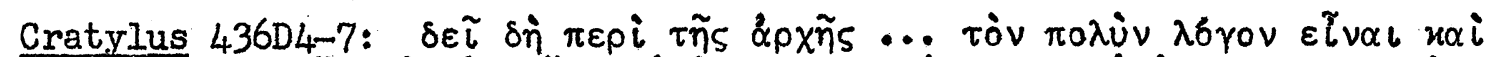

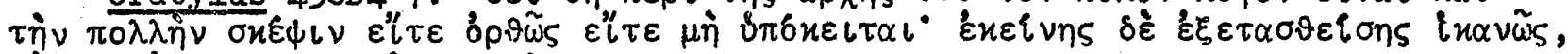

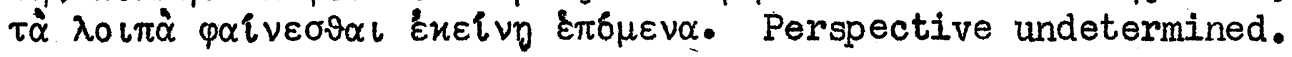

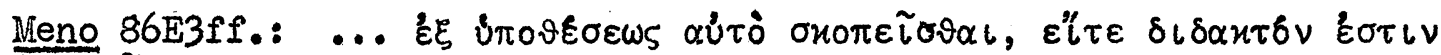

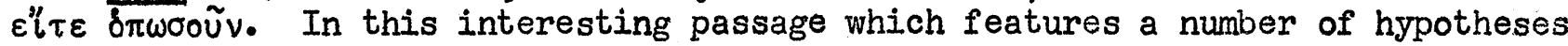
in action, with the result that all of them are either demonstrated or refuted or both, the perspective is undetermined throughout.

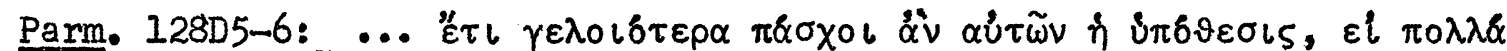

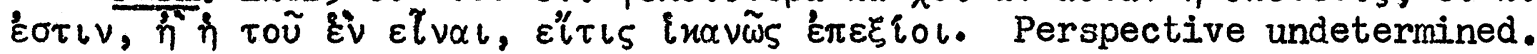

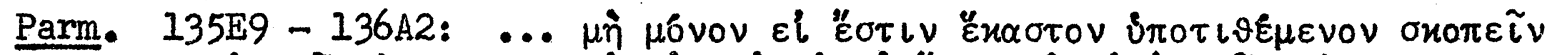

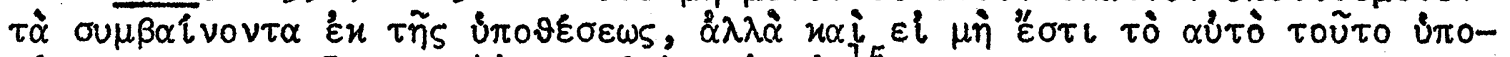
$\tau\{\vartheta \varepsilon \sigma \vartheta \alpha \iota . .$. Perspective undetermined. 15

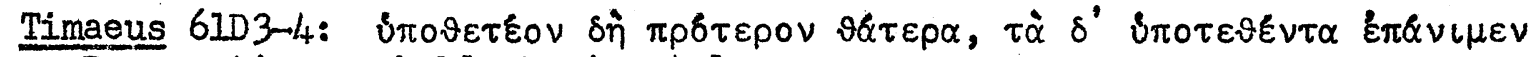
$\alpha$ ง เ५. Perspective probably horizontal.

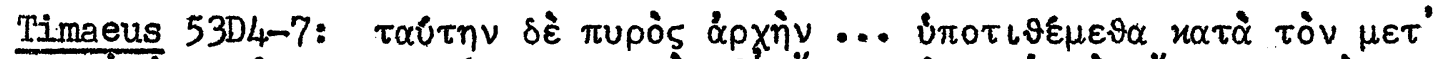

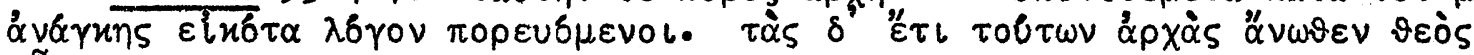
oi $\delta \varepsilon . . .$. This last sentence poses a problem on which neither Taylor nor Cornford comments. The question is whether " $\alpha \omega \vartheta \vartheta v v$ goes with the preceding

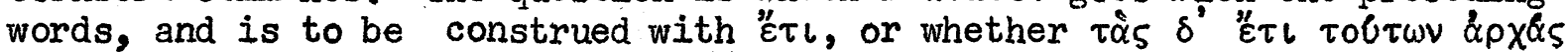
forms a self-contained unit, as Archer-Hind certainly understood it, in which case "ै $\nu \omega \vartheta \varepsilon v$ signifies the position from which the god exercises his knowledge. If the latter interpretation is adopted, as I suspect it should, " $\nu \omega \vartheta \varepsilon v . .$. olde would be parallel to such an expression as Theaet. 175D $3 \beta \lambda \varepsilon \pi \omega \nu . .$.

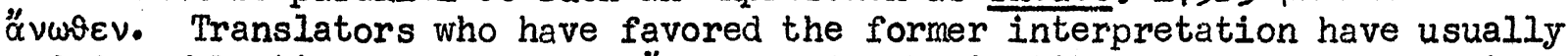
ended up blunting the force of $\alpha \nu \omega \vartheta \varepsilon \nu$ and substituting innocuous terms like "remote" or "principal." - Hence, probably, perspective undetermined.

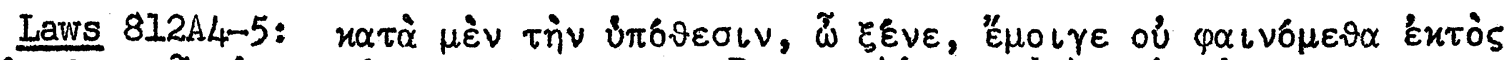
$\pi \circ \rho \varepsilon \delta \varepsilon \sigma \vartheta \alpha \iota \tau \tilde{\omega} \nu$ $\delta \pi 0 \tau \varepsilon \vartheta \varepsilon \nu \tau \omega \nu \lambda \delta \gamma \omega \nu . .$. Perspective undetermined. 


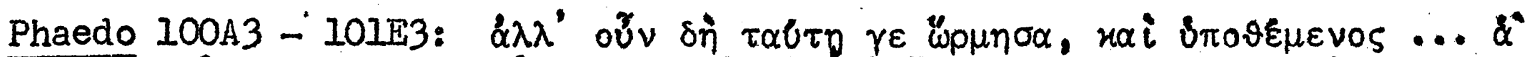

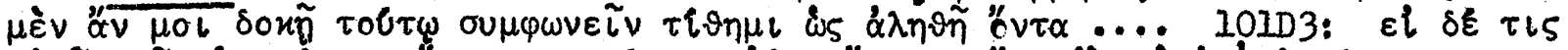

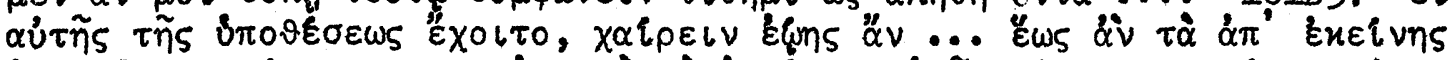

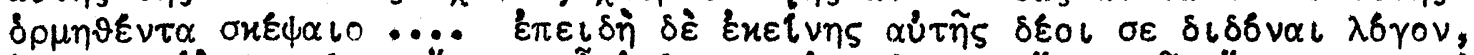

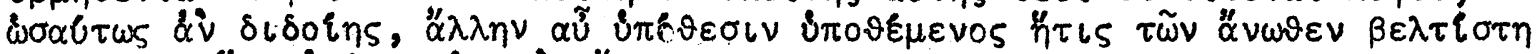

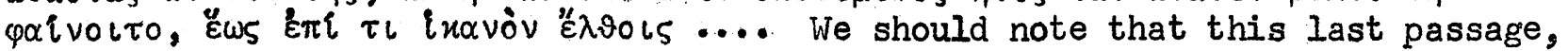
as so many others dealing with hypothesis, employs a verb of motion or progression, in this case $\delta \rho \mu \alpha \omega$ and $\delta \rho \mu \alpha o \mu \alpha \iota$. The usual sense of the word is "to move forward," in a horizontal direction. The direction may occasionally be vertical; but in that case it is likely to be upward rather than downward, for the simple reason that the word denotes willed rather than automatic motion.

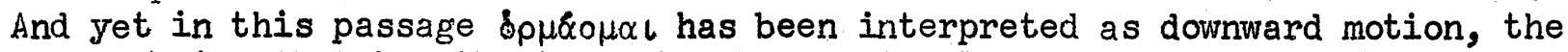
reason being that hypothesis in the Phaedo is clearly associated with the Ideas, and the Ideas are highest. However, the argument is not from Ideas to sensibles and back but, according to Socrates, from Ideas to Ideas; and there is no evidence to indicate, at least in this passage, that one Idea is higher than another. Thus the more comprehensive or basic hypothesis should not be pictured as lying above a hypothesis entailed by it or generated by it. Never

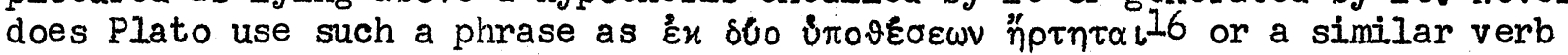
of suspension in connexion with the hypothesis method. But we can go further than that, and suggest that in the Phaedo Plato seems to conceive of the premise as lying below the conclusion, that is to say, that plato adopts the

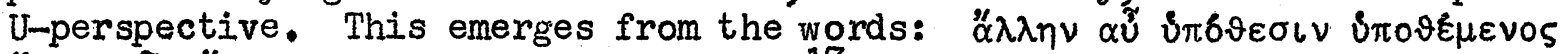

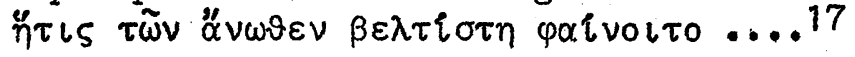

Plato's language here points to the notion of an inverted pyramid, with the more comprehensive hypothesis lying at the bottom, and the $\delta p \mu \eta \vartheta \varepsilon v \tau \alpha$

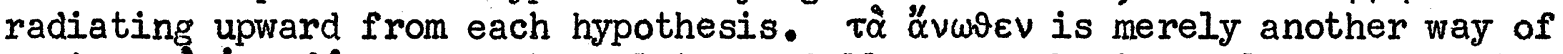
saying $\tau \alpha \dot{~} \delta \mu \eta \eta \vartheta \varepsilon \tau \tau$. We translate as follows: "placing below hypothesis X another hypothesis $Y$ which would seem to be the best (hypothesis) of the (propositions) above it," that is, which would account best for X and other statements on the same level. Usually the genitive $\tau \tilde{\omega} \nu ~ \alpha \nu \nu \omega \vartheta \varepsilon \nu$ is explained as a partitive genitive. It is, however, equally possible to take it as an objective genitive, referring to the conclusions generated by the premise. Cf. the Aristotelian parallel given above, note 17. The word $\beta \varepsilon \lambda \tau\{\sigma \tau \eta$ in the sense of "most effective" may seem unusual, but the functional connotation of

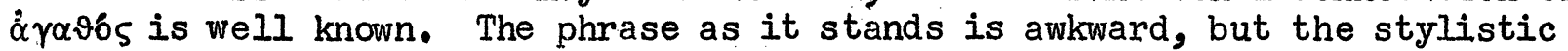
difficulties seem to me less decisive than the difficulties of interpretation which result from the traditional assumption of a "higher hypothesis." To imply, as one would have to on the old assumption, that there are many hypotheses of a more universal character, any of which might be relevant to the argument, is to render the method itself almost unworkable. The plural $\tau \tilde{\omega} v$ makes better sense if it can be supposed to refer to the several conclusions inferrable from a particular premise chosen with the assistance of common sense and synoptic experience.18

If we now draw the balance of the passages we have discussed, it appears that most of them do not tell us anything about the direction of the activity prompted by hypothesis. Two, perhaps three instances favor the horizontal perspective; here the eye focusses on the progress of the operation rather than on the mutual relations between the terms of the operation. One passage

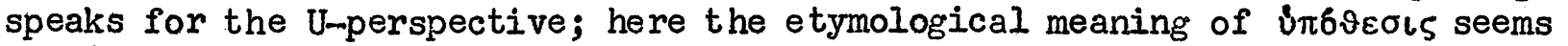
to rise more fully to the consciousness. And finally, there is no evidence of the A-perspective. 
It now remains to investigate some passages from the Republic, particularly from the section containing the allegory of the Cave and the diagram of the Divided Line. These passages have been reserved for the final part of our discussion because they appear, at first glance, to occasion the greatest difficulty. It is only natural, given the perspectival connotations of Cave and Line, that the Platonic distinction between "up" and "down" should here find its most marked expression. We should, however, remember that this perspective refers to the distinction between levels of reality, or rather between reality and the various kinds of non-real, and to the distinction between the correlate mental activities, such as knowledge and belief. The method of hypothetical reasoning, on the other hand, functions on only one level of mental activity, and is concerned with only one level of reality, viz., the Ideas $(511(1)-2)$, whether these Ideas be visualized in their pure state, as in dialectic, or less purely, as in mathematics and other sciences ( $510 \mathrm{~B} 4 \mathrm{ff}$.).

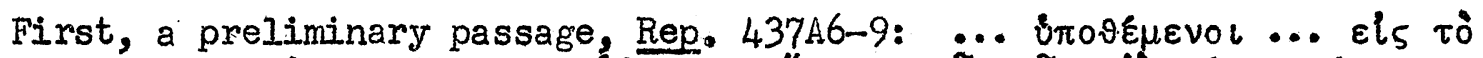

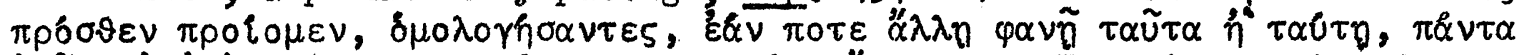

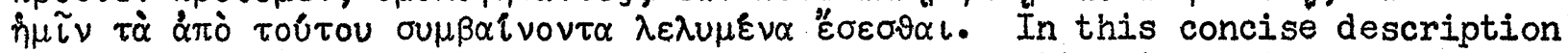
of argument on the basis of postulates, the perspective is horizontal, or at best undetermined. What makes the passage important is the fact that the quotations to be studied directly refer by and large to just such reasoning as is contemplated here, in language very similar to the terms used here.

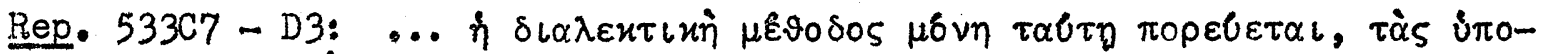

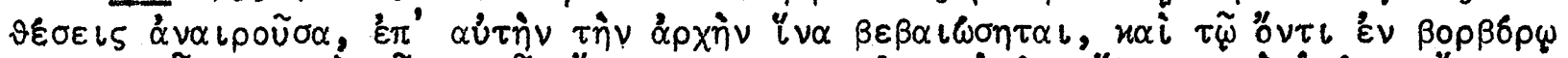

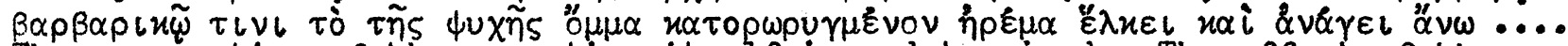
The perspective of the operation itself is undetermined. The effect of the operation is to turn the mind upward from the swamp of Becoming to the exalted status of Reality. Analogously, the activity of dialectic is seen to lie at

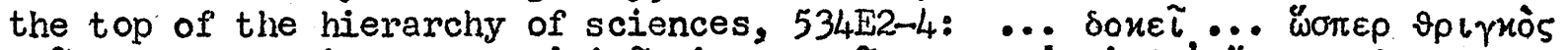

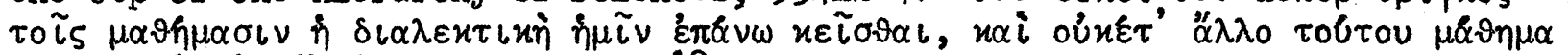

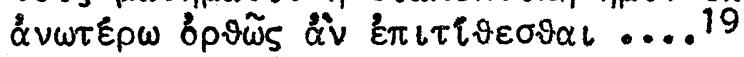

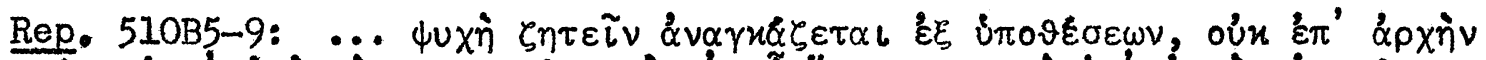

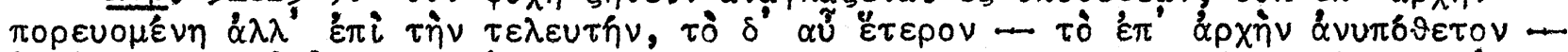

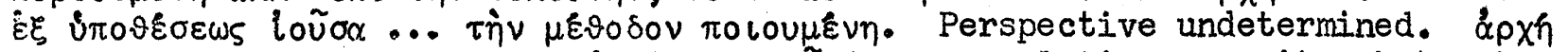

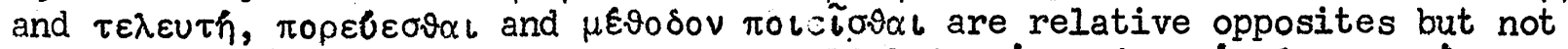

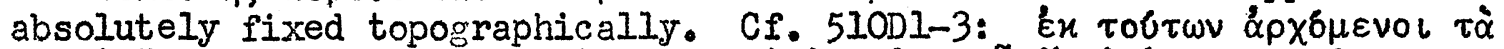

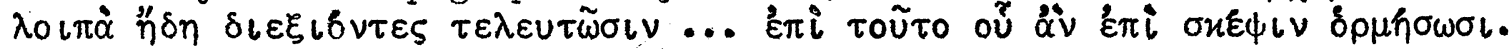

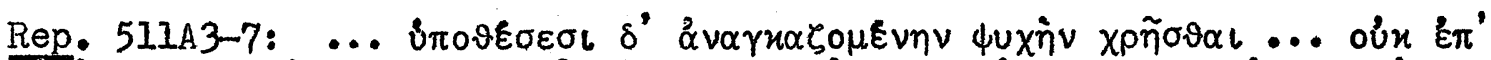

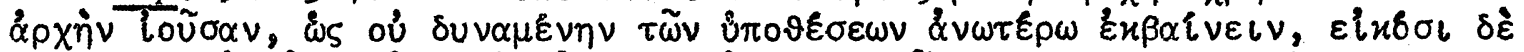

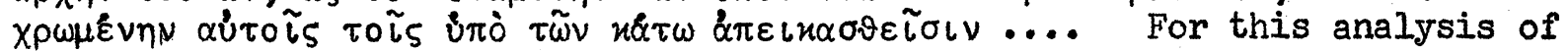
non-dialectic hypothetical argument, cfo above, 510B5-9. There is, however, one difference between this statement and other statements we have discussed:

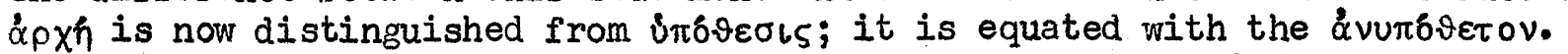
Mathematical demonstration is contrasted with the genuine dialectical method

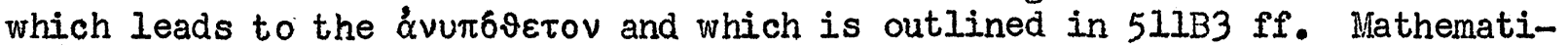
cal reasoning functions on the second highest level of mental activity. Compared with pure dialectic, it is a swamp which does not permit a man to get his head free. That is to say, in the hierarchy of the sciences 
mathematics is so far below dialectic that it might as well be thought to be located in the quagmire of belief. There is no transfer or transition possible between mathematical and dialectical reasoning. "To get above the (mathematical) hypotheses" is tantamount to entering an entirely different arena of logical endeavor.

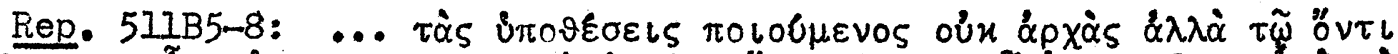

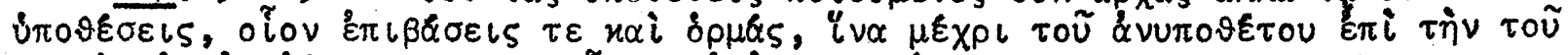

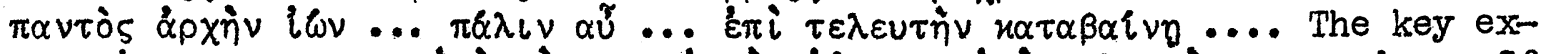

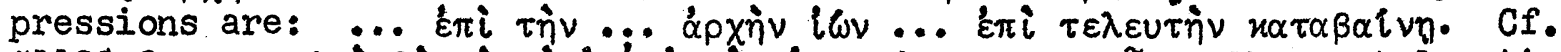

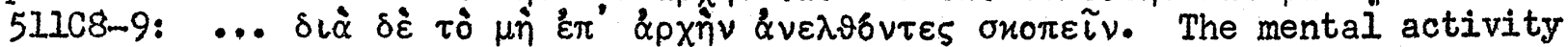
described is that of dialectic, as contrasted with that of mathematics and the inferior sciences. It is difficult to decide whether the words, and expecially the prepositions employed in the compound verbs, refer to a vertical or a horizontal perspective. The horizontal or "progress of argument" significance of $\dot{\alpha} v \dot{\alpha}-$ and $x \alpha \tau \dot{\alpha}-c o m p o u n d s$ is well known and has already been in-

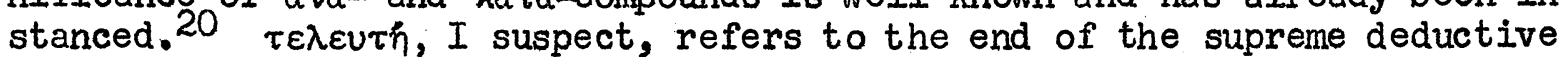
argument, just as ápx refers to its beginning, or to the end of the ensuing synthesis. What complicates the situation is the image, probably humorous, of the stepping stones, which seem to indicate an "up" and "down" orientation. And yet, if that were Plato's conscious intention, he might well have spoken of higher and lower hypotheses, or he might have detailed the location of the 'pxh vis-a-vis the other "steps" in the logical process. This he does not do. For the rest, the language does not differ significantly from the language of other passages we have discussed. The best we can say, therefore, is that for one moment there is promise of a vertical perspective coming out into the open, only to be silenced immediately by what is probably a vague assumption of horizontal procedure. Whether the vertical perspective lurking under the surface would have been a U-perspective, as is suggested by the image of the stepping stones, or an A-perspective, as the alternative meaning of the prepositions may indicate, is impossible say. But this very uncertainty should help to drive home the point that Plato is not in this context interested in establishing an unambiguous vertical perspective.

To repeat, when Plato, in his analysis of the Divided line and Cave,

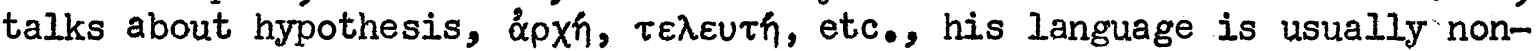
committal on the score of perspective. Whe re he does speak of "up" and "down" he is ranking scientific hypotheses in the second highest division, below those of dialectic. Once, $511 \mathrm{~B} 5 \mathrm{ff.}$, the ontological and epistemological context does, on one interpretation, seem to color the logical perspective, and he appears to believe that those hypotheses which bring a man closer to the

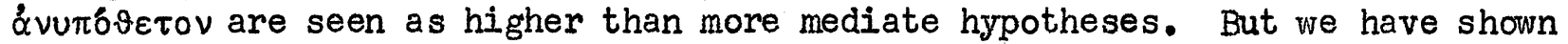
that the language need not carry this meaning, and that the similar phrasing of other passages speaks against the adoption of this interpretation.

We conclude, therefore, that the evidence for Plato regarding the argum ment by hypothesis as leading downward from premise to inference is nil. It may be wondered why Plato was misunderstood so soon, notably by Aristotle. One explanation may be that Plato provides few examples of the technique in action. Another may be that Plato's followers regarded him as a metaphysician first and last, and could not separate his logic from his ontology. The problem calls for a close investigation.

To be read at the west coast meeting of the Society for incient Greek Philoscohy in Claremont, California, November 1958. 
NOTES

1) R. Robinson, Plato's Earlier Dialectic ${ }^{2}$ (Oxford 1953) 136, ch. 9 \#4.

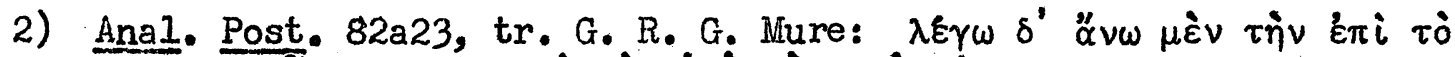

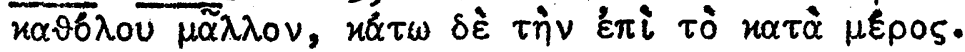

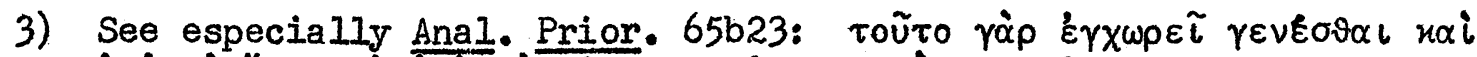

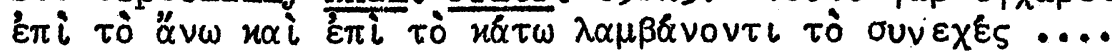

4) Cf. the passage just preceding the footnote on terminology, Anal. Post.

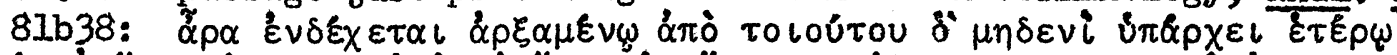

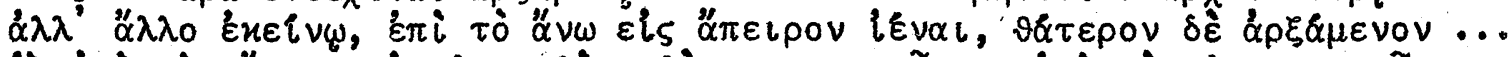

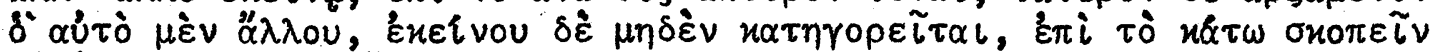

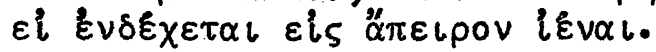

5) Anal. Post. 97a33.

6) Met. 992a18.

7) I wish to thank Professors J. B. Skemp and D. J. Allan for reading an earlier draft of the ms, and suggesting many valuable changes. Niy gratitude does not not, however, imply that they approve the thesis of this paper.

8) C. Ritter, "Platons Logik," Philologus 75 (1919) I ff., and passim.

9) That "subsumption" is regularly visualized by Plato as containment, appears also from such passages as Gorgias 464. $\mathrm{ff}$., and Theaet.

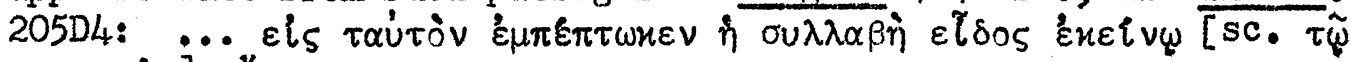

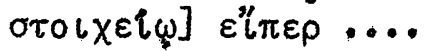

10) The word is Robinson's, p. 95.

11) Robinson, po 112 .

12) J. Burnet, ed, Plato's Euthyphro etc. (Oxford 1924) 51.

13) Robinson, pp, 68 and 98.

14) It may be asked how the notion of "dependence" is naturally expressed in Greek. The word "dependence" Itself, of course, betrays an A-perspective; here the more solid or more substantial matter is pictured at the top. In Greek also this perspective is utilized for the notion of dependence and vital connexion, chiefly through the verb $\alpha \rho \tau \alpha \omega$ and its compound $\alpha$ $\alpha \alpha \rho \tau \alpha \omega$. Herodotus, for example, uses them frequently in this sense. But more or less the same conception may be expressed via the verb $x \varepsilon i \tilde{\mu} \alpha \mathrm{l}$ in combination with the preposition $\varepsilon v$; and there the perspective is the opposite. Thus the linguistic data bearing on the notion of "dependence" are inconclusive on the score of perspective. 
15) In this passage, $\delta \pi 6 \vartheta \varepsilon \sigma \iota \varsigma$ has come to mean little more than $\pi \rho 6 \tau \alpha \sigma \iota 5$; this becomes clear from the fact that in the sequel the various hypotheses are $\varepsilon l-$ clauses. - Robinson, pp. 278-280, indicates the ways in which hypothesis in the Parmenides differs from the hypothetical method in the Republic. One difference on which he comments, with apparent surprise: "There is almost no trace in the Parm. of the upward path of the Rep." Incorrect; there is no trace whatever. Furthermore, as we shall see later, there probably is no upward path in the Republic either.

16) Olympiod. in Phaed. 188 line 3 Norvin.

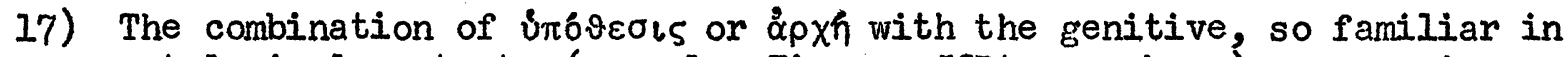
ontological contexts (example: Timaeus 53D4, as above), recurs in

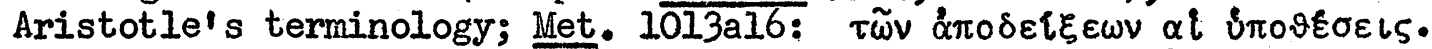

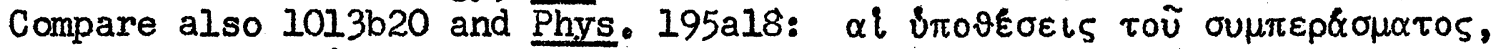
where however $\alpha i \tau i \alpha$ is understood.

18) R. S. Bluck, in Phronesis 2 (1957) 26 asks what Plato could have meant by the "higher" hypothesis that is to be substituted for the hypothesis that turned out to be unsatisfactory. He believes that Plato is here thinking of the Form of the Good, i.e., the teleological cause. He shrugs off the stubborn fact that Socrates had announced he was going to undertake a $\delta \varepsilon \delta \tau \varepsilon p \circ \varsigma \pi \lambda \circ \tilde{s}$. On rejecting the translation "higher hypothesis" the difficulty resolves itself.

19) F. M. Cornford's translation of Rep. $510 \mathrm{~B} 4 \mathrm{ff}$. and $533 \mathrm{C} 7 \mathrm{ff}$. freely interpolates "up" and "down," instead of reproducing the inconclusive perspective of the text. H. D. P. Lee has managed to do this admirably.

20) Cf. $\varepsilon \alpha \alpha \eta \tilde{r} \varepsilon v$, Xenophon Memor. 4.6.13, cited above, page 4; also the

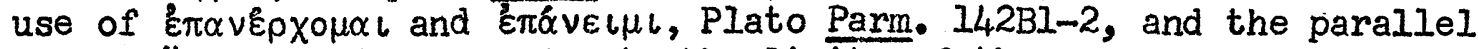
use of " $\alpha \nu \omega$ and $u \& \tau \omega$ to refer to the limits of the race course, Plato Rep. 613BII-12. Cf. further 0. Becker, Das Bild des Weges, Hermes

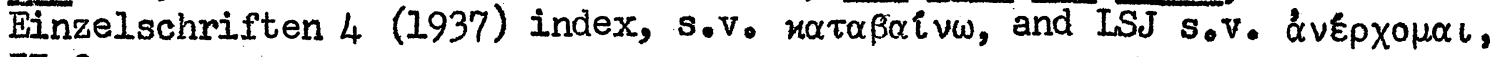
II.2. 